\title{
Proton-Helium Elastic Electromagnetic Cross-Section
}

\author{
Burn Huang and King Y. Ng* \\ Institute of Modern Physics, Chinese Academy of Sciences \\ 509 Nanchang Road, Lanzhou, China 730000
}

(November, 2015)

\begin{abstract}
In the test facility of the C-ADS project, ${ }^{\dagger} \mathrm{A} 25-\mathrm{MeV}$ proton beam is directed to hit a target consisting of 1-mm tungsten balls lubricated by $100-\mathrm{Pa}$ helium gas. To estimate the power loss to the helium gas, an accurate collision cross section is computed.
\end{abstract}

*Fermi National Accelerator Laboratory, PO Box 500 Batavia, IL 60510, USA.

${ }^{\dagger}$ Conceptual Physics Design on the C-ADS Accelerators, IHEP-CADS-Report/2012-01E, Ed. Jingyu Tang and Zhihui Li, August, 2012, Institute of High Energy Physics, CAS, Institute of Modern Physics, CAS. 


\title{
Proton-Helium Elastic Electromagnetic Cross-section
}

\author{
Burn Huang and King Y. Ng* \\ Institute of Modern Physics, Chinese Academy of Sciences \\ 509 Nanchang Road, Lanzhou, China 730000
}

(November, 2015)

\begin{abstract}
In the test facility of the $\mathrm{C}-\mathrm{ADS}$ project [1], A $25-\mathrm{MeV}$ proton beam is directed to hit a target consisting of 1-mm tungsten balls lubricated by $100-\mathrm{Pa}$ helium gas. To estimate the power loss to the helium gas, an accurate collision cross section is computed.
\end{abstract}

\section{Introduction}

A high energy transport line (HEPT) is designed for the prototype linac of the CADS project. A $10-\mathrm{mA} 25-\mathrm{MeV}$ proton beam from the superconducting linac will pass through this HEBT to hit the dense granular target, which consists of 1-mm diameter tungsten balls lubricated by $100-\mathrm{Pa}$ helium gas. It is important to estimate the power losses as a result of the protonhelium interaction [2]. Since the relevant proton kinetic energy is low, nuclear reaction can be neglected. In this note, we will derive the elastic scattering cross section between the incident proton beam and the target helium nuclei according to quantum electrodynamics. The result will be used to estimate the power loss of the proton beam to the helium gas.

\section{Fermion-Boson Electromagnetic Interaction}

Here we are using the natural units commonly employed in quantum field theory; i.e., velocity of light $c=1$ and Planck constant divided by $2 \pi \hbar=1$. The Lagrangian density describing

\footnotetext{
${ }^{*}$ Fermi National Accelerator Laboratory, PO Box 500 Batavia, IL 60510, USA.
} 
the free proton field $\psi$, the free helium field $\varphi$, and the free photon field $F_{\mu \nu}=\partial_{\mu} A_{\nu}-\partial_{\nu} A_{u}$ is

$$
\mathcal{L}_{0}=-\bar{\psi}\left(\gamma_{\mu} \partial_{\mu}+m\right) \psi-\left(\partial_{\mu} \varphi^{*} \partial_{\mu} \varphi+M^{2} \varphi^{*} \varphi\right)-\frac{1}{4} F_{\mu \nu} F_{\mu \nu}
$$

where $m$ and $M$ are, respectively, the rest masses of the proton and helium. Here $\gamma_{\mu}$ are the $4 \times 4$ Dirac matrices, $\psi$ is a $4 \times 1$ matrix, and $\bar{\psi}=\psi^{\dagger} \gamma_{4}$ is a $1 \times 4$ matrix. The first term of the Lagrangian density in Eq. (2.1) leads to the free Dirac equation for proton, the second term leads to the free Klein-Gordon equation for helium, while the third term leads to the sourceless Maxwell equation. Electromagnetic interactions are introduced by

$$
\partial_{\mu} \psi \rightarrow\left(\partial_{\mu}-i e A_{\mu}\right) \psi \quad \text { and } \quad \partial_{\mu} \varphi \rightarrow\left(\partial_{\mu}-i q A_{\mu}\right) \varphi
$$

respectively, for proton of charge $e$ and helium of charge $q$. The interaction Lagrangian density is therefore

$$
\mathcal{L}_{1}=i e \bar{\psi} \gamma_{\mu} \psi A_{u}-i q\left(\varphi^{*} \partial_{\mu} \varphi-\partial_{\mu} \varphi^{*} \varphi\right) A_{u}
$$

where the term of $\mathcal{O}\left(q^{2}\right)$, which is of higher order, has been neglected. ${ }^{\dagger}$

We study here the scattering of a proton by a helium particle. The kinematic is illustrated in Fig. 3.1(a), where the proton has initial 4 -momentum ${ }^{\ddagger} p_{i}=\left(\vec{p}_{i}, i E_{i}\right)$ and final 4-momentum $p_{f}=\left(\vec{p}_{f}, i E_{f}\right)$. The initial and final helium 4-momenta are, respectively, $k_{i}=\left(\vec{k}_{i}, i \varepsilon_{i}\right)$ and $k_{f}=\left(\vec{k}_{f}, i \varepsilon_{f}\right)$. We work in the center-of-mass frame. Therefore $\vec{p}_{i}+\vec{k}_{i}=0$, $\vec{p}_{f}+\vec{k}_{f}=0$. Since the scattering is elastic, the magnitudes of the proton initial and final 3 -momenta as well as the magnitudes of the helium initial and final 3-momenta are the same. We denote them as $p=\left|\vec{p}_{i}\right|=\left|\vec{p}_{f}\right|=\left|\vec{k}_{i}\right|=\left|\vec{k}_{f}\right|$. For the energy, we have $E_{i}=E_{f}$, and $\varepsilon_{i}=\varepsilon_{f}$. To ease writing, we remove the subscripts wherever possible and denote them simply by $E$ and $\varepsilon$.

\section{Feymann Graph and Feymann Rules [3]}

There is only one Feymann graph for the interaction, which is depicted in Fig. 3.1(b), We list briefly the Feymann rules. Only those relevant for the Feymann graph in Fig. 3.1(b) are included:

\footnotetext{
${ }^{\dagger}$ It is important to point out that the proton charge $e$ (or $q$ ) that appears in Eq. (2.2) above is defined as $e=\sqrt{4 \pi \hbar c / \alpha}$ or $e=\sqrt{4 \pi / \alpha}$ when $\hbar=1$ and $c=1$, where $\alpha \approx 1 / 137$ is the fine-structure constant. To arrive at the SI units, it should be multiplied by $\sqrt{4 \pi \epsilon_{0}}$ again.

${ }_{\ddagger}^{\ddagger}$ The metric $\delta_{\mu \nu}$ with $\mu, \nu=1, \cdots, 4$ is used.
} 


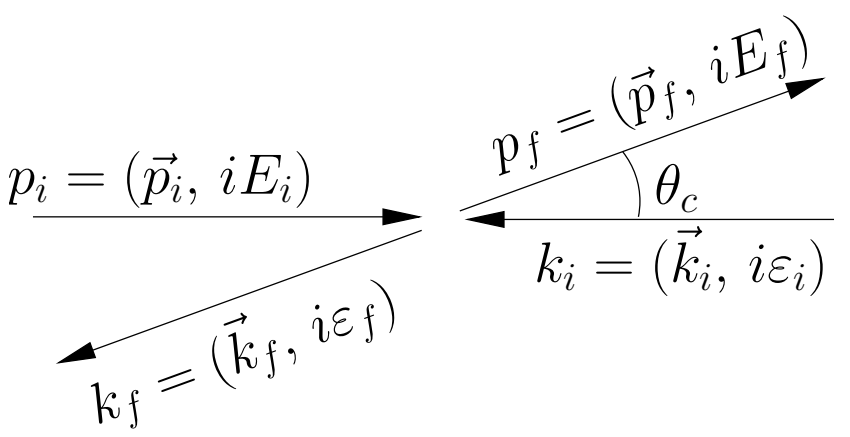

(a)

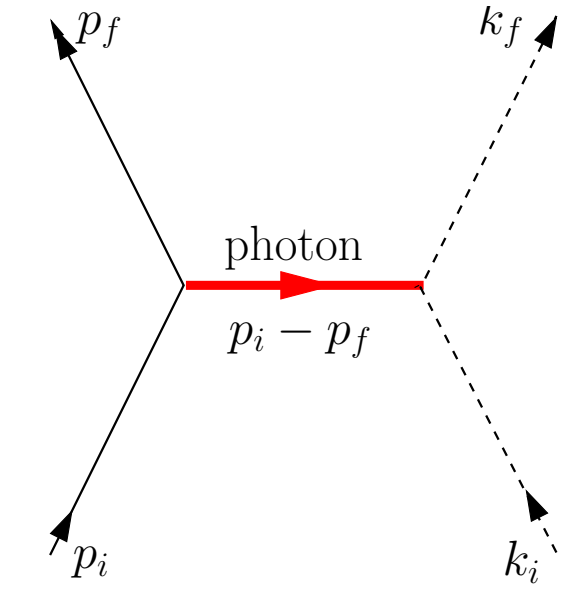

(b)

Figure 3.1: (a) Scattering of a proton by a helium particle in the center-of-mass frame. (b) The Feymann graph representing the scattering.

1. Incoming and outgoing proton: $u_{i}$ and $u_{f}^{\dagger}$.

2. Incoming and outgoing helium: $\frac{1}{\sqrt{2 \varepsilon_{i} V}}$ and $\frac{1}{\sqrt{2 \varepsilon_{f} V}}$.

3. Photon propagator: $\frac{-i \delta_{\mu \nu}}{\left(k_{f}-k_{i}\right)^{2}-i \epsilon}$ with $\epsilon$ positive and infinitesimal.

4. Proton-photon vertex: $-e \gamma_{\mu}$.

5. Helium-photon vertex: $i q\left(k_{i}+k_{f}\right)_{\nu}$.

The helium field are expanded in the momentum-space within a finite volume $V$. The coefficients $a_{k}$ and $a_{k}^{\dagger}$ are normalized and quantized so that they represent the annihilation and creation operators of the helium particle. What are left, $1 / \sqrt{2 \varepsilon_{i, f} V}$, are the wavefunctions. The proton field is treated in the same way and $u_{i, f}$ are the wavefunctions and are $4 \times 1$ matrices. Thus both $u_{i, f}$ are inversely proportional to $\sqrt{V}$.

\section{Scattering Matrix}

The scattering matrix $S$ represents the number of interactions taken place within the volume $V$ in the time period $T$. It can be written as

$$
\left\langle p_{f}, k_{f}|S| p_{i}, k_{i}\right\rangle=(2 \pi)^{4} \delta^{4}\left(p_{i}+k_{i}-p_{f}-k_{f}\right) \mathcal{M}_{f i}
$$


The interaction matrix $\mathcal{M}$ is formulated using the above Feymann rules:

$$
\mathcal{M}_{f i}=\bar{u}_{f}\left(-e \gamma_{\mu}\right) u_{i} \frac{-i \delta_{\mu \nu}}{\left(k_{f}-k_{i}\right)^{2}-i \epsilon} \frac{i q\left(k_{i}+k_{f}\right)_{\nu}}{\sqrt{4 \varepsilon_{i} V \varepsilon_{f} V}} .
$$

Considering that fact that the evaluation is in the center-of-mass frame, $\left(k_{f}-k_{i}\right)^{2}=$ $4 p^{2} \sin ^{2} \frac{\theta_{c}}{2}$, where $p=\left|\vec{p}_{i}\right|=\left|\vec{p}_{f}\right|$ and $\theta_{c}$ is the scattered angle. The above can be simplified as

$$
\mathcal{M}_{f i}=-\frac{e q u_{f}^{\dagger} \gamma_{4}\left(\hat{k}_{i}+\hat{k}_{f}\right) u_{i}}{8 \varepsilon V p^{2} \sin ^{2} \frac{\theta_{c}}{2}}
$$

where the abbreviation $\hat{k}=k_{\mu} \gamma_{\mu}$ has been used. The $i \epsilon$ part in the denominator of the interaction matrix has been omitted, because we are working with a finite scattered angle $\theta_{c}$. We are interested in the absolute value squared of the interaction or $S$ matrix. Here there is only one Feymann graph; we therefore need not pay too much attention to its sign or whether the phase at each element of the Feymann graph. Taking the absolute value squared,

$$
\left|\mathcal{M}_{f i}\right|^{2}=\frac{e^{2} q^{2} \mathcal{R}}{64 \varepsilon^{2} V^{2} p^{4} \sin ^{4} \frac{\theta_{c}}{2}}
$$

where

$$
\mathcal{R}=\operatorname{Tr}\left(\hat{k}_{i}+\hat{k}_{f}\right) u_{i} \bar{u}_{i} \gamma_{4}\left(\hat{k}_{i}^{\dagger}+\hat{k}_{f}^{\dagger}\right) \gamma_{4} u_{f} \bar{u}_{f}
$$

Notice that $\gamma_{\mu} \gamma_{4}=-\gamma_{4} \gamma_{\mu}$ for $\mu=1,2,3$. When $\mu=4$, remembering that $k_{\mu}^{*}=-k_{\mu}$. we therefore have $\gamma_{4} \hat{k}^{\dagger} \gamma_{4}=-\hat{k}$. As a fermion, the proton has two spin states. Since we are not interested in the spin states, we should average over the initial proton spin states and sum over the final proton spin states. We have then

$$
\begin{aligned}
\mathcal{R} & =-\frac{1}{2} \operatorname{Tr}\left(\hat{k}_{i}+\hat{k}_{f}\right) \sum_{i} u_{i} \bar{u}_{i}\left(\hat{k}_{i}+\hat{k}_{f}\right) \sum_{f} u_{f} \bar{u}_{f} \\
& =-\frac{1}{2} \operatorname{Tr}\left(\hat{k}_{i}+\hat{k}_{f}\right) \frac{-i \hat{p}_{i}+m}{2 E_{i} V}\left(\hat{k}_{i}+\hat{k}_{f}\right) \frac{-i \hat{p}_{f}+m}{2 E_{f} V} \\
& =-\frac{1}{8 E_{i}^{2} V^{2}} \operatorname{Tr}\left(\hat{k}_{i}+\hat{k}_{f}\right)\left(-i \hat{p}_{i}+m\right)\left(\hat{k}_{i}+\hat{k}_{f}\right)\left(-i \hat{p}_{f}+m\right),
\end{aligned}
$$

where we have used the fact that,

$$
\sum_{i} u_{i}\left(\vec{p}_{i}\right) \bar{u}_{i}\left(\vec{p}_{i}\right)=\frac{-i \hat{p}_{i}+m}{2 E_{i} V} \text { and } \sum_{f} u_{f}\left(\vec{p}_{f}\right) \bar{u}_{f}\left(\vec{p}_{f}\right)=\frac{-i \hat{p}_{f}+m}{2 E_{f} V}
$$


The trace in Eq. (4.6) can be easily computing. Setting $k_{s}=k_{i}+k_{f}$, we have ${ }^{\S}$

$$
\begin{aligned}
\mathcal{R} & =\frac{1}{8 E_{i}^{2} V^{2}} \operatorname{Tr}\left[\hat{k}_{s}\left(\hat{p}_{i}+i m\right) \hat{k}_{s}\left(\hat{p}_{f}+i m\right)\right] \\
& =\frac{1}{8 E^{2} V^{2}} \operatorname{Tr}\left[\hat{k}_{s} \hat{p}_{i} \hat{k}_{s} \hat{p}_{f}-m^{2} \hat{k}_{s} \hat{k}_{s}+i m\left(\hat{k}_{s} \hat{p}_{i} \hat{k}_{s}+\hat{k}_{s} \hat{k}_{s} \hat{p}_{f}\right)\right] \\
& =\frac{4}{8 E^{2} V^{2}}\left[2\left(k_{s} \cdot p_{i}\right)\left(k_{s} \cdot p_{f}\right)-\left(k_{s} \cdot k_{s}\right)\left(p_{i} \cdot p_{f}\right)-m^{2}\left(k_{s} \cdot k_{s}\right)\right],
\end{aligned}
$$

where $(A \cdot B)$ denotes the dot product of two 4 -vectors $A$ and $B$. We have

$$
\begin{aligned}
\left(k_{s} \cdot k_{s}\right) & =-4\left(M^{2}+p^{2} \sin ^{2} \frac{\theta_{c}}{2}\right), \\
\left(p_{i} \cdot p_{f}\right)+m^{2} & =-2 p^{2} \sin ^{2} \frac{\theta_{c}}{2} \\
\left(k_{s} \cdot p_{i}\right)=\left(k_{s}, p_{f}\right) & =-2 p^{2} \cos ^{2} \frac{\theta_{c}}{2}-2 E \varepsilon .
\end{aligned}
$$

Then

$$
\begin{aligned}
\mathcal{R} & =\frac{4}{E^{2} V^{2}}\left\{\left(p^{2} \cos ^{2} \frac{\theta_{c}}{2}+E \varepsilon\right)^{2}-p^{2} \sin ^{2} \frac{\theta_{c}}{2}\left(M^{2}+p^{2} \sin ^{2} \frac{\theta_{c}}{2}\right)\right\} \\
& =\frac{4}{E^{2} V^{2}}\left\{\left(p^{2}+E \varepsilon\right)^{2}-p^{2} \sin ^{2} \frac{\theta_{c}}{2}\left[(E+\varepsilon)^{2}-m^{2}\right]\right\} .
\end{aligned}
$$

The absolute value squared of the interaction matrix then becomes

$$
\left|\mathcal{M}_{f i}\right|^{2}=\frac{e^{2} q^{2}}{16 E^{2} \varepsilon^{2} V^{4} p^{4} \sin ^{4} \frac{\theta_{c}}{2}}\left\{\left(p^{2}+E \varepsilon\right)^{2}-p^{2} \sin ^{2} \frac{\theta_{c}}{2}\left[(E+\varepsilon)^{2}-m^{2}\right]\right\} .
$$

We see that $\mathcal{R}$ assumes a minimum at back scattering or when $\theta_{c}=\pi$. At this moment

$$
\mathcal{R}=\frac{4 m^{2} \varepsilon^{2}}{E^{2} V^{2}}
$$

which is definitely non-negative, which serves as a partial check of our derivation.

\section{Differential Cross-Section}

The absolute value squared of the $S$ matrix denotes the total number of interactions or events in time $T$ and volume $V$. It is therefore related to the scattering cross-section $\sigma$ as

$$
\sum_{\vec{p}_{f} \vec{k}_{f}}\left|\left\langle p_{f}, k_{f}|S| p_{i}, k_{i}\right\rangle\right|^{2}=\frac{\sigma|\Delta v| T}{V},
$$

§The easily proven formulas, $\operatorname{Tr}\left(\gamma_{\mu} \gamma_{\nu}\right)=4 \delta_{\mu \nu}, \operatorname{Tr}\left(\gamma_{\mu} \gamma_{\nu} \gamma_{\rho}\right)=0$, and $\operatorname{Tr}\left(\gamma_{\mu} \gamma_{\nu} \gamma_{\rho} \gamma_{\sigma}\right)=4\left(\delta_{\mu \nu} \delta_{\rho \sigma}+\delta_{\mu \sigma} \delta_{\nu \rho}-\right.$ $\left.\delta_{\mu \rho} \delta_{\nu \sigma}\right)$ have been used. 
where $\Delta v$ is the initial relative velocity of the two particles under consideration. For one incoming particle in the interaction volume, the density is $1 / V$. Thus the right side of Eq. (5.1) represents the number of particles in the volume $V$ going into the cross-sectional area $\sigma$ in time $T$. When the summation is over all final states, the result is the total crosssection. When the summation is only partial, the differential cross-section results. We have for the total cross-section,

$$
\sigma=\sum_{\vec{p}_{f} \vec{k}_{f}} \frac{(2 \pi)^{8} V}{|\Delta v| T} \delta^{4}\left(p_{i}+k_{i}-p_{f}-k_{f}\right) \delta^{4}\left(p_{i}+k_{i}-p_{f}-k_{f}\right)\left|\mathcal{M}_{f i}\right|^{2} .
$$

There are two 4-dimensional $\delta$-functions and they are exactly the same. The argument of one of them can be set to zero. Since

$$
\frac{1}{(2 \pi)^{4}} \int e^{i p x} d^{4} x=\delta^{4}(p)
$$

we can write $(2 \pi)^{4} \delta^{4}(0)=V T$, the 4 -dimensional space-time volume. The cross-section then becomes

$$
\sigma=\sum_{\vec{p}_{f} \vec{k}_{f}} \frac{(2 \pi)^{4} V^{2}}{|\Delta v|} \delta^{4}\left(p_{i}+k_{i}-p_{f}-k_{f}\right)\left|\mathcal{M}_{f i}\right|^{2} .
$$

For finite interaction volume $V$, the final states are discrete. When $V$ is extended to infinity, the summation over $\vec{p}_{f}$ and $\vec{k}_{f}$ becomes integrals. The transformation is

$$
\frac{1}{V^{2}} \sum_{\vec{p}_{f} \vec{k}_{f}} \rightarrow \int \frac{d^{3} p_{f}}{(2 \pi)^{3}} \frac{d^{3} k_{f}}{(2 \pi)^{3}}
$$

and therefore

$$
\sigma=\frac{2 \pi}{|\Delta v|} \int \frac{d^{3} p_{f} d^{3} k_{f}}{(2 \pi)^{3}} \delta^{4}\left(p_{i}+k_{i}-p_{f}-k_{f}\right)\left|V^{2} \mathcal{M}_{f i}\right|^{2}
$$

As we can see, the interaction volume $V$ gets cancelled out as it should be. For this reason, one may leave out $V$ or set it as unity from the very beginning. We can readily integrate over $d^{3} k_{f}$ using the momentum $\delta$-functions, leaving behind the energy $\delta$-function. Next we write $d^{3} p_{f}=p_{f}^{2} d p_{f} d \Omega$ and try to integrate over $d p_{f}$ using the energy $\delta$-function, where $p_{f}=\left|\vec{p}_{f}\right|$ and equals $p$ that we defined before. The total final energy is $E_{t}=\sqrt{p_{f}^{2}+m^{2}}+\sqrt{p_{f}^{2}+M^{2}}$. Thus

$$
\frac{d E_{t}}{d p_{f}}=\frac{p_{f}\left(E_{f}+\varepsilon_{f}\right)}{E_{f} \varepsilon_{f}},
$$

and

$$
\int d^{3} p_{f} \delta\left(E_{t}-E_{i}-\varepsilon_{i}\right)=\int p_{f}^{2} d \Omega \frac{d p_{f}}{d E_{t}} d E_{t} \delta\left(E_{t}-E_{i}-\varepsilon_{i}\right)=\int \frac{p \varepsilon E}{\varepsilon+E} d \Omega .
$$


The relative velocity is

$$
|\Delta v|=\frac{p_{i}}{E_{i}}+\frac{p_{i}}{\varepsilon_{i}}=\frac{p(E+\varepsilon)}{E \varepsilon} .
$$

Putting everything together the differential cross-section is

$$
\frac{d \sigma}{d \Omega}=\frac{e^{2} q^{2}}{4 \pi^{2}} \frac{\left(p^{2}+E \varepsilon\right)^{2}-p^{2} \sin ^{2} \frac{\theta_{c}}{2}\left[(E+\varepsilon)^{2}-m^{2}\right]}{16(E+\varepsilon)^{2} p^{4} \sin ^{4} \frac{\theta_{c}}{2}} .
$$

Since we obtain the differential cross-section already, we wish to express it in the SI units, something that we are familiar with. " At the same time, we generalize the scattering by letting the charge of the incident particle be $e Z_{i}$ and its rest mass by $m=A_{i} m_{p}$ with $m_{p}$ being the proton rest mass. For the charged target particle, we write $q=Z_{t} e$. We arrive at

$$
\frac{d \sigma}{d \Omega}=\left(\frac{e^{2} Z_{i} Z_{t}}{4 \pi \epsilon_{0}}\right)^{2} \frac{\left(p^{2} c^{2}+E \varepsilon\right)^{2}-p^{2} c^{2} \sin ^{2} \frac{\theta_{c}}{2}\left[(E+\varepsilon)^{2}-m^{2} c^{2}\right]}{4(E+\varepsilon)^{2} p^{4} c^{4} \sin ^{4} \frac{\theta_{c}}{2}} .
$$

Introducing the proton classical radius $r_{p}=e^{2} /\left(4 \pi \epsilon_{0} m_{p} c^{2}\right)$, the cross-section becomes

$$
\frac{d \sigma}{d \Omega}=\left(\frac{Z_{i} Z_{t} r_{p}}{A_{i}}\right)^{2} \frac{\left(p^{2} c^{2}+E \varepsilon\right)^{2}-p^{2} c^{2} \sin ^{2} \frac{\theta_{c}}{2}\left[(E+\varepsilon)^{2}-m^{2} c^{2}\right]}{4(E+\varepsilon)^{2} \gamma_{i}^{2} \beta_{i}^{2} p^{2} c^{2} \sin ^{4} \frac{\theta_{c}}{2}},
$$

where $\gamma_{i}$ and $\beta_{i}$ are the relativistic parameters of the incident particle (the proton) in the center-of-mass frame. The first factor has the dimension of area. The second factor is dimensionless with the familiar $\sin ^{4} \frac{\theta_{c}}{2}$ in the denominator.

The proton is scattered into the walls of the beam pipe and will be lost if the scattered angle $\left|\theta_{c}\right|>\theta_{b c}$. This critical loss angle $\theta_{b c}$ will be derived later below. Thus for the loss cross-section, we need to integrate $d \Omega$ over the polar angle from $\theta_{b c}$ to $\pi$ and the azimuthal angle $\phi$ from 0 to $2 \pi$, or

$$
\sigma_{\text {loss }}=2 \pi\left(\frac{Z_{i} Z_{t} r_{p}}{A_{i}}\right)^{2} \int_{\theta_{b c}}^{\pi} \sin \theta_{c} d \theta_{c} \frac{\left(p^{2} c^{2}+E \varepsilon\right)^{2}-p^{2} c^{2} \sin ^{2} \frac{\theta_{c}}{2}\left[(E+\varepsilon)^{2}-m^{2} c^{2}\right]}{4(E+\varepsilon)^{2} \gamma_{i}^{2} \beta_{i}^{2} p^{2} c^{2} \sin ^{4} \frac{\theta_{c}}{2}},
$$

We will be dealing with the integrals

$$
\int_{\theta_{b c}}^{\pi} \frac{\sin \theta_{c} d \theta_{c}}{\sin ^{4} \frac{\theta_{c}}{2}}=\int_{\theta_{b c}}^{\pi} \frac{4 d \sin \frac{\theta_{c}}{2}}{\sin ^{3} \frac{\theta_{c}}{2}}=\frac{2}{\tan ^{2} \frac{\theta_{b c}}{2}} \text { and } \int_{\theta_{b c}}^{\pi} \frac{\sin \theta_{c} d \theta_{c}}{\sin ^{2} \frac{\theta_{c}}{2}}=\int_{\theta_{b c}}^{\pi} \frac{4 d \sin \frac{\theta_{c}}{2}}{\sin \frac{\theta_{c}}{2}}=-2 \ln \sin ^{2} \frac{\theta_{b c}}{2} \text {. }
$$

The final result is

$$
\sigma_{\text {loss }}=\pi\left(\frac{Z_{i} Z_{t} r_{p}}{A_{i}}\right)^{2}\left\{\frac{\left(p^{2} c^{2}+E \varepsilon\right)^{2}}{(E+\varepsilon)^{2} \gamma_{i}^{2} \beta_{i}^{2} p^{2} c^{2} \tan ^{2} \frac{\theta_{b c}}{2}}+\frac{\left[(E+\varepsilon)^{2}-m^{2} c^{2}\right] \ln \sin ^{2} \frac{\theta_{b c}}{2}}{(E+\varepsilon)^{2} \gamma_{i}^{2} \beta_{i}^{2}}\right\} .
$$

\footnotetext{
IThe conversion consists of $p \rightarrow p c, m \rightarrow m c^{2}$, and $e^{2} \rightarrow 4 \pi e^{2} /\left(4 \pi \epsilon_{0}\right)=e^{2} / \epsilon_{0}$.
} 


\section{Comparison with Classical Rutherford Scattering For- mula}

The scattering is studied in two special limits: one with the mass of the target very heavy or $M \gg m$ and one in the non-relativistic regime.

\subsection{Very Heavy Target}

When the mass of the target is very heavy, its energy $\varepsilon$ dominates over $E$ and $p c$. Equation (5.12) reduces to

$$
\frac{d \sigma}{d \Omega}=\left(\frac{Z_{i} Z_{t} r_{p}}{A_{i}}\right)^{2} \frac{1-\beta_{i}^{2} \sin ^{2} \frac{\theta_{c}}{2}}{4 \gamma_{i}^{2} \beta_{i}^{4} \sin ^{4} \frac{\theta_{c}}{2}} .
$$

Since the target is infinitely heavy, the scattering is equivalent to a scattering by a central field supplied by the target. Under this condition, the center-of-mass frame is the same as the lab frame where the target is stationary. Thus the angle $\theta_{c}$ by which the incident particle is scattered is the same scattered angle in the lab frame. However, when compared with the Rutherford scattering cross-section of a relativistic particle by a central field, we find an extra term $\beta_{i}^{2} \sin ^{2} \frac{\theta_{c}}{2}$ in the numerator. The presence of the extra term comes from the fact that the incident particle is a fermion with two spin states. Equation (6.1) is also known as Mott scattering cross-section [4].

\subsection{Non-Relativistic Regime}

In the non-relativistic limit, $E \rightarrow m c^{2}, \varepsilon \rightarrow M c^{2}, \gamma_{i} \rightarrow 1, \gamma_{f} \rightarrow 1, p / m c \rightarrow 0$, and $p / M \rightarrow 0$. Our notation exhibits a subscript $\ell$ for quantities in the lab frame and a subscript $c$ for quantities in the center-of-mass frame. The subscript $c$ is often dropped as was in earlier Sec. 1-4.

The scattering differential cross-section of Eq. (5.12) becomes

$$
\frac{d \sigma}{d \Omega}=\left(\frac{Z_{i} Z_{t} r_{p}}{A_{i}}\right)^{2} \frac{m^{2} M^{2}-m^{2} \beta_{i}^{2} \sin ^{2} \frac{\theta_{c}}{2}\left[(m+M)^{2}-m^{2}\right]}{4(m+M)^{2} \beta_{i}^{4} m^{2} \sin ^{4} \frac{\theta_{c}}{2}} .
$$

We wish to express the cross-section in terms of the relative velocity $\beta_{\mathrm{rel}} c$ between the two 
particles. From Eq. (5.9), we obtain

$$
\beta_{\mathrm{rel}} c=|\Delta v|=\frac{p(m+M)}{m M}=\frac{\beta_{i} c(m+M)}{M}=\frac{\beta_{i} c m}{\mu},
$$

where we have introduced the reduced mass $\mu$ for the 2-particle system, which is defined as

$$
\mu=\frac{m M}{m+M} \quad \text { or } \quad \frac{1}{\mu}=\frac{1}{m}+\frac{1}{M} .
$$

The differential cross-section becomes

$$
\frac{d \sigma}{d \Omega}=\left(\frac{Z_{i} Z_{t} r_{p} m}{A_{i} \mu}\right)^{2} \frac{1-\beta_{\mathrm{rel}}^{2} \sin ^{2} \frac{\theta_{c}}{2}\left[1-\mu^{2} / M^{2}\right]}{4 \beta_{\mathrm{rel}}^{4} \sin ^{4} \frac{\theta_{c}}{2}} .
$$

Introduce the classical radius of a particle of charge $e$ with reduced mass $\mu$ as

$$
r_{\mu}=\frac{e^{2}}{4 \pi \epsilon_{0} \mu c^{2}}=r_{p} \frac{m}{A_{i} \mu}
$$

The differential cross-section can now be written as

$$
\frac{d \sigma}{d \Omega}=\frac{\left(Z_{i} Z_{t} r_{\mu}\right)^{2}}{4 \beta_{\mathrm{rel}}^{4} \sin ^{4} \frac{\theta_{c}}{2}}\left[1-\beta_{\mathrm{rel}}^{2} \sin ^{2} \frac{\theta_{c}}{2}\left(1-\frac{\mu^{2}}{M^{2}}\right)\right] .
$$

The first term is the Rutherford Coulomb-scattering differential cross-section of a particle of mass $m$ by another particle of mass $M$ in the non-relativistic limit with the reduce mass substituted. The second term comes from the fermion nature of the incident particle. However, actually this term should have been dropped in the non-relativistic regime because it is of $\mathcal{O}\left(\beta_{\text {rel }}^{2}\right)$.

\section{Connection with the Lab Frame}

The differential cross-section of Eq. (5.12) and the loss cross-section of Eq. (5.15) are derived in the center-of-mass frame. Since they count the number of interaction events, they are invariant when Lorentz transformed in the direction of the incident particles. Thus Eqs. (5.12) and (5.15) remain unchanged in the lab frame, provided that quantities such as momenta, energies, and scattered angle in the center-of-mass frame are substituted. In this section, we are going to derive relation of these quantities as functions of their lab-frame values. 


\subsection{Lorentz Transformation}

We denote the incident proton as particle 1 and the target helium as particle 2. To avoid confusion, we denote all quantities in the center-of-mass frame by the additional subscript $c$ and those quantities in the lab frame by the additional subscript $\ell$. Thus all the relativistic parameters in Eqs. (5.12) and (5.15) such as $\gamma_{i}$ and $\beta_{i}$ should be replaced by $\gamma_{c 1 i}$ and $\beta_{c 1 i}$. Notice that another subscript, the particle number, has been added to specify which particle they describe.

In the lab frame, particle 1 with initial 3-momentum $\vec{p}_{\ell i}$ is head-on towards particle 2 which is at rest. It is scattered by the angle $\theta_{\ell}$ and its 3 -momentum becomes $\vec{p}_{\ell f}$. The helium particle with $\vec{k}_{\ell i}=0$ will recoil to the 3 -momentum $\vec{k}_{\ell f}$. We choose $z$ as the direction $\vec{p}_{\ell i}$ and the scattering takes place in the $x-z$ plane as shown in Fig. 7.2. First, let us compute the relativistic parameters $\vec{\beta}_{t r}$ and $\gamma_{t r}$ that boost the lab frame to the center-of-mass frame. Obviously $\vec{\beta}_{t r}$ is in the negative $z$-direction and have the value $\beta_{t r}=\beta_{c 2 i}$, the velocity of particle 2 or the helium in the center-of-mass frame. Thus the velocity of particle 1 is transformed from $\beta_{\ell 1 i}$ in the lab frame to $\beta_{c 1 i}$ in the center-of-mass frame via

$$
\beta_{c 1 i}=\frac{\beta_{\ell 1 i}-\beta_{t r}}{1-\beta_{t r} \beta_{\ell 1 i}}
$$

The 4-vector square of the total 4-momentum, which is an invariant in any frame, is, when

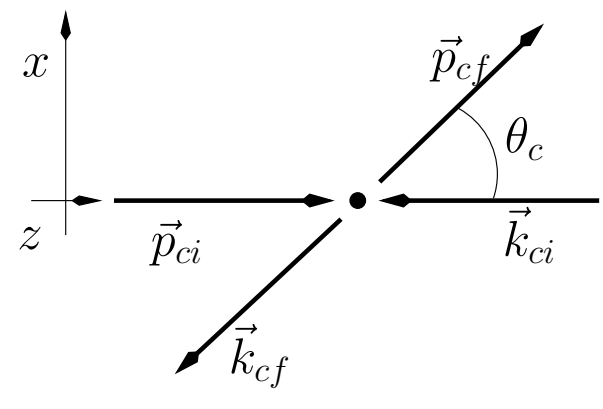

(a)

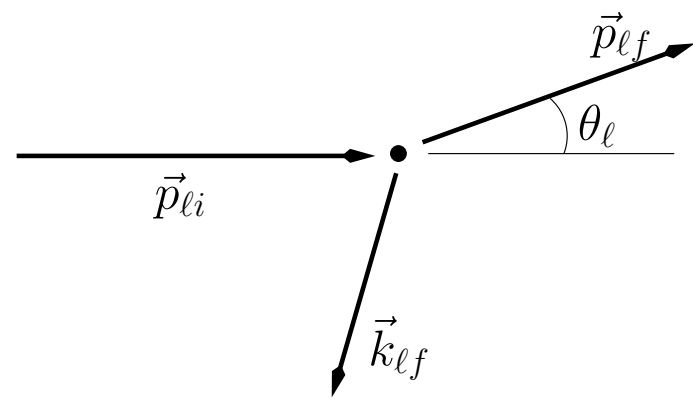

(b)

Figure 7.2: (a) Center-of-mass (COM) frame with subscript $c$ and (b) lab frame with subscript $\ell$. In lab frame, particle 1 (proton) with momentum $\vec{p}_{\ell i}$ is head-on towards particle 2 (helium) which is at rest. Particle 1 is scattered to momentum $\vec{p}_{\ell f}$ at the scattered angle $\theta_{\ell}$, while particle 2 recoils with momentum $\vec{k}_{\ell f}$. In the COM frame, all corresponding momenta are denoted by subscript $c$, and the scattered angle becomes $\theta_{c}$. 
evaluated in the center-of-mass frame,

$$
\begin{aligned}
\sqrt{-\left(p_{c i}+k_{c i}\right)_{\mu}\left(p_{c i}+k_{c i}\right)_{\mu}} & =\gamma_{c 1 i} m+\gamma_{c 2 i} M=\frac{\gamma_{c 1 i} m \beta_{c 1 i}}{\beta_{c 1 i}}+\gamma_{c 2 i} M \\
& =\gamma_{c 2 i} M\left(1+\frac{\beta_{c 2 i}}{\beta_{c 1 i}}\right)=\gamma_{c 2 i} M\left(1+\frac{\beta_{t r}}{\beta_{c 1 i}}\right) \\
& =\gamma_{c 2 i} M\left(1+\frac{\beta_{t r}\left(1-\beta \beta_{\ell 1 i}\right)}{\beta_{c 1 i}-\beta_{t r}}\right)
\end{aligned}
$$

where for the third equality, use has been made by the fact that $\gamma_{c 1 i} m \beta_{c 1 i}=\gamma_{c 2 i} M \beta_{c 2 i}$ in the center-of-mass frame. We have set $c=1$ for the sake of convenience. When evaluated in the lab frame, we get

$$
-\left(p_{\ell i}+k_{\ell i}\right)_{\mu}\left(p_{\ell i}+k_{\ell i}\right)_{\mu}=\left(\gamma_{\ell 1 i} m+M\right)^{2}-\left(\gamma_{\ell 1 i} \beta_{\ell 1 i} m\right)^{2}=m^{2}+M^{2}+2 \gamma_{\ell 1 i} m M .
$$

Equating the two evaluations, the Lorentz boost $\beta_{t r}$ can be solved as a function of $\beta_{\ell 1 i}$, the velocity of the incoming proton in the lab frame.

If we denote the total energy of the system in the center-of-mass frame by the mass $\mathcal{M}$ of a composite particle,

$\mathcal{M}^{2}=-\left(p_{\ell i}+k_{\ell i}\right)_{\mu}\left(p_{\ell i}+k_{\ell i}\right)_{\mu}=\left(E_{\ell i}+M\right)^{2}-\vec{p}_{\ell i}^{2}=\left(E_{\ell 1 i}+M\right)^{2}-\left(E_{\ell 1 i}^{2}-m^{2}\right)=m^{2}+M^{2}+2 M E_{\ell 1 i}$.

Then transforming to the lab frame, this composite particle has the energy $\gamma_{t r} \mathcal{M}$, where $\gamma_{t r}=1 / \sqrt{1-\beta_{t r}^{2}}$ is the Lorentz transformation $\gamma$. We have then $\gamma_{t r} \mathcal{M}=E_{\ell i}+M$. We therefore have the Lorentz transformation

$$
\gamma_{t r}=\frac{E_{\ell i}+M}{\mathcal{M}}=\frac{E_{\ell i}+M}{m^{2}+M^{2}+2 M E_{\ell i}} .
$$

In the lab frame, the composite particle has the 3 -momentum $\gamma_{t r} \mathcal{M} \beta_{t r}$ in the $z$-direction. This will the the same as $\vec{p}_{\ell i}$. Thus

$$
\vec{\beta}_{t r}=-\frac{\vec{p}_{\ell i}}{\gamma_{t r} \mathcal{M}}=-\frac{\vec{p}_{\ell i}}{E_{\ell i}+M}
$$

The negative sign comes about because $\vec{p}_{\ell i}$ points to the positive $z$-direction while $\vec{\beta}_{t r}$ points to the negative $z$-direction. Thus, from the momentum $\vec{p}_{\ell i}$ and energy $E_{\ell i}$ of the incident proton in the lab frame, the Lorentz boost $\vec{\beta}_{t r}$ from the lab frame to the center-of-mass frame can be computed using Eq. (7.6). The incident velocity of the proton in the centerof-mass frame $\beta_{c 1 i}$ can now be computed using Eq. (7.1). The velocity of the target helium in the center-of-mass frame is just $\beta_{c 2 i}=\beta_{t r}$. From these, the energies and momenta in the center-of-mass frame are therefore known. 


\subsection{Scattered Angle}

Now come to the scattered angle. In the lab frame, when a proton in the particle beam is kicked transversely by the angle $\theta_{\ell}$ at a location where the betatron function is $\beta_{\ell}$, the transverse amplitude at another location $B$ is

$$
x=\theta_{\ell} \sqrt{\beta_{\ell} \beta_{B}} \sin \Delta \phi
$$

where $\beta_{B}$ is the betatron function at location $B$ and $\Delta \phi$ is the betatron phase advance from the position of the kick to location $B$. If location $B$ happens to be at a point where the transverse aperture is at a minimum radius $b$, the proton will be lost if

$$
\theta_{\ell} \sqrt{\beta_{\ell} \beta_{B}} \gtrsim b
$$

Averaging over the beam pipe where electromagnetic scattering of the proton by helium particles will take place, we obtain the maximum angle

$$
\theta_{\ell}=\frac{b}{\sqrt{\beta_{B} \beta_{a v}}}
$$

above which the scattered protons will shoot over the aperture and will be lost. Here $\beta_{a v}$ is the betatron function averaged over the part of the beam pipe of concern. The next task is to relate $\theta_{\ell}$ in the lab frame with $\theta_{c}$ in the center-of-mass frame employed in Eqs. (5.12) and (5.15).

For the proton in the center-of-mass frame, the Lorentz transformation of the $z$-component momentum and energy of the proton (or particle 1) reads

$$
p_{c i z}=\gamma_{t r}\left(p_{\ell i z}-\beta_{t r} E_{\ell i}\right), \quad E_{c i}=\gamma_{t r}\left(E_{\ell i}-\beta_{t r} p_{\ell i z}\right),
$$

where we have written out the component directions as subscripts for clarification. The momentum component of this particle in the $z$ and $x$ directions and the particle energy are, respectively,

$$
p_{c f x}=p_{c i z} \sin \theta_{c}, \quad p_{c f z}=p_{c i z} \cos \theta_{c}, \quad p_{c f 0}=p_{c i 0}=E_{c i},
$$

where the subscript 0 indicate the $4^{\text {th }}$ component of the 4 -vector with $i$ removed. The transformation back to the lab frame is the same Lorentz transformation but with velocity $-\beta_{t r}$. Hence

$$
\begin{aligned}
& p_{\ell f x}=p_{c f x}=p_{c i z} \sin \theta_{c}, \\
& p_{\ell f z}=\gamma_{t r}\left(p_{c f z}+\beta_{t r} p_{c f 0}\right)=\gamma_{t r}\left(p_{c i z} \cos \theta_{c}+\beta_{t r} E_{c i}\right), \\
& p_{\ell f 0}=\gamma_{t r}\left(p_{c f 0}+\beta_{t r} p_{c f z}\right)=\gamma_{t r}\left(E_{c i}+\beta_{t r} p_{c i z} \cos \theta_{c}\right) .
\end{aligned}
$$


The relation between the scattered angles in the two frames is therefore

$$
\tan \theta_{\ell}=\frac{p_{\ell f x}}{p_{\ell f z}}=\frac{\sin \theta_{c}}{\gamma_{t r}\left(\cos \theta_{c}+\beta_{t r} E_{c i} / p_{c i z}\right)}=\frac{\sin \theta_{c}}{\gamma_{t r}\left(\cos \theta_{c}+\beta_{t r} / \beta_{c i}\right)},
$$

from which, we can solve

$$
\theta_{c}=\sin ^{-1} \frac{\gamma_{t r} \beta_{t r} \tan \theta_{\ell}}{\beta_{c i} \sqrt{1+\gamma_{t r}^{2} \tan ^{2} \theta_{\ell}}}+\tan ^{-1}\left(\gamma_{t r} \tan \theta_{\ell}\right)
$$

\section{Application to CADS}

A high energy transport line (HEBT) is designed for the prototype linac of the CADS project. A $10-\mathrm{mA} 25-\mathrm{MeV}$ proton beam from the superconducting linac will pass through this HEBT to hit the dense granular target, which consists of 1-mm diameter tungsten balls lubricated by $100-\mathrm{Pa}$ helium gas. It is important to estimate the power losses as a result of the proton-helium Coulomb interaction.

For the proton-helium system, the relevant parameters are listed in Table I.

Table I: The relativistic parameters of proton and helium as well as the loss angle in the lab frame and the center-of-mass frame.

\begin{tabular}{|l|c|c|c|c|}
\hline & \multicolumn{2}{|c|}{ Proton } & \multicolumn{2}{c|}{ Helium } \\
\hline Mass & \multicolumn{2}{|c|}{1} & \multicolumn{2}{c|}{4} \\
Charge & \multicolumn{2}{|c|}{1} & $\beta_{\ell 2, c 2}$ & $\gamma_{\ell 2, c 2}$ \\
\hline & $\beta_{\ell 1, c 1}$ & $\gamma_{\ell 1, c 1}$ & 0 & 1 \\
\hline Lab frame & 0.2264 & 1.0266 & 0.04623 & 1.00107 \\
COM frame & 0.1820 & 1.0170 & 0.010 .2283 & \\
Relative & 0.2283 & & -0.221 \\
\hline Loss angle $(\mathrm{mr})$ & \multicolumn{3}{|c|}{$\theta_{b \ell}=4.924, \theta_{b c}=6.181$} \\
\hline
\end{tabular}

The loss cross-section of Eq. (5.15) can be rewritten as

$$
\sigma_{\text {loss }}=\pi\left(\frac{Z_{i} Z_{t} r_{p}}{A_{i}}\right)^{2}\left\{\frac{\left(\gamma_{c 1} \beta_{c 1}^{2}+\gamma_{c 2} M / m\right)^{2}}{\left(\gamma_{c 1}+\gamma_{c 2} M / m\right)^{2} \gamma_{c 1}^{2} \beta_{c 1}^{4} \tan ^{2} \frac{\theta_{b c}}{2}}+\left[1-\frac{1}{\left(\gamma_{c 1}+\gamma_{c 2} M / m\right)^{2}}\right] \frac{\ln \sin ^{2} \frac{\theta_{b c}}{2}}{\gamma_{c 1}^{2} \beta_{c 2}^{2}}\right\}
$$


which gives $\sigma_{\text {loss }}=1.765 \times 10^{-27} \mathrm{~m}^{2}$. The second term amounts to only $0.0011 \%$ of the first and is completely unimportant.

A $25-\mathrm{MeV}$ proton in the lab frame can be considered non-relativistic. In such limit, Eq. (6.7) leads to the loss cross-section

$$
\sigma_{\text {loss }}=\pi \frac{\left(Z_{i} Z_{t} r_{\mu}\right)^{2}}{\beta_{\text {rel }}^{4} \tan ^{2} \frac{\theta_{b c}}{2}},
$$

where the $r_{\mu}=r_{p}(m+M) / M$ is the classical particle radius corresponding to the reduced mass. This gives $\sigma_{\text {loss }}=1.784 \times 10^{-27} \mathrm{~m}^{2}$, which is about $1 \%$ larger than using the nonreduced formula for the cross-section. In other words, because of the non-relativistic nature of the problem, the Rutherford scattering cross-section applies. However, one must pay careful attention that (1) the classical particle radius corresponds to that of the reduced mass, (2) the factor $\beta^{4}$ in the denominator corresponds to the relative velocity in the centerof-mass frame, and (3) the loss angle corresponds to the angle $\theta_{b c}$ in the center-of-mass frame.

The power loss is computed as follows. The density of helium particle in

$$
n=\frac{P}{k T},
$$

where $P=100 \mathrm{~Pa}$ or 100 newton- $\mathrm{m}^{2}, T=300^{\circ} \mathrm{K}$ is the helium temperature, and $k=$ $1.3807 \times 10^{-23}$ joules $/ \mathrm{K}$ is the Boltzmann constant. The power loss is given by

$$
\frac{d P_{\text {beam }}}{d x}=\frac{\sigma_{\text {loss }} n E I}{e}=16.0 \mathrm{~W} / \mathrm{m}
$$

where $e$ is the proton charge, $E=25 \mathrm{MeV}$ is the kinetic energy of the proton beam, and $I=10 \mathrm{~mA}$ is the beam current.

As the proton energy increases, the relative velocity between the proton and helium increases and eventually reaching the limit $\beta_{\text {rel }}=2$. Keeping the loss angle $\theta_{\ell}$ constant at $4.92 \mathrm{mrad}$, the loss angle increases in the center-of-mass frame. The result is that the loss cross-section and power loss decrease rapidly. This is illustrated in Fig. 8.3. Notice that so far only electromagnetic interaction has been assumed in the scattering. In the wave aspect of quantum mechanic, a particle with momentum $p$ is equivalent to a wave with wavelength

$$
\lambda=\frac{h}{p},
$$


where $h$ is the Planck constant with $\hbar c=197.3 \mathrm{MeV}$-fm. When $\lambda \lesssim 2 \mathrm{fm}$, nuclear-force effect will become important. This will occur when the proton kinetic energy reaches $\sim 310 \mathrm{MeV}$. In other words, the illustration in Fig. 8.3 will be correct because nuclear-force effects will be negligibly small. Hoowever, in Phase II and Phase III of the C-ADS project where the proton kinetic energy reaches, respectively, $1 \mathrm{GeV}$ and $1.5 \mathrm{GeV}$, nuclear effects must be included in the loss analysis.

\section{References}

[1] Conceptual Physics Design on the C-ADS Accelerators, IHEP-CADS-Report/2012-01E, Ed. Jingyu Tang and Zhihui Li, August, 2012, Institute of High Energy Physics, CAS, Institute of Modern Physics, CAS.

[2] Huan Jia, Yuan He, Youjin Yuan, Shuhui Liu, Chenzhang Yuan, Peng Zhang, Yuanshuai Qin, and Zhijun Wang, Beam Dynamics Design of the HEBT for CADS, submitted to Nuclear Instruments and Methods in Physics Research Section A, 2015.

[3] R.P. Feymann, Phys. Rev. 75, 486, 1736 (1949), 76, 749, 769 (1949); J.D. Bjorken and S.D. Drell, Relativistic Quantum Mechanics, McGraw Hill, 1964,

[4] N.F. Mott, Proc. Roy. Soc. (London), A124, 425 (1929). 

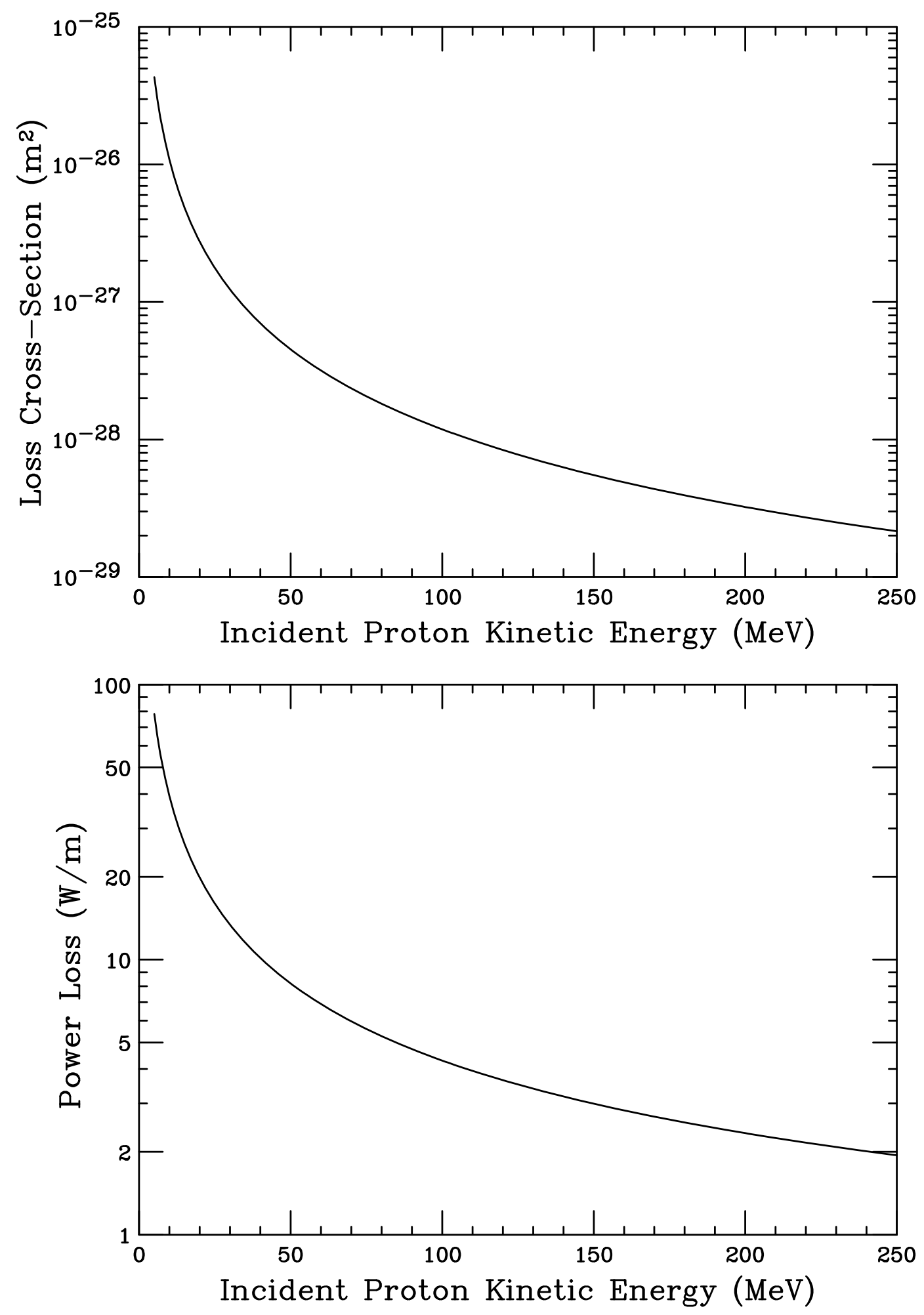

Figure 8.3: Top: Loss cross-section for a proton beam with helium particles as a function of the proton kinetic energy. Only electromagnetic interaction is assumed. The loss angle in the lab frame is kept at $\theta_{\ell}=4.92 \mathrm{mr}$. Bottom: The corresponding power loss of the proton beam as a function of proton kinetic energy. The helium pressure and temperature are kept at $100 \mathrm{~Pa}$ and $300 \mathrm{~K}^{\circ}$, while the proton beam current is at $10 \mathrm{~mA}$. 